\title{
La responsabilidad social como estrategia de inclusión laboral para el desarrollo de las organizaciones públicas en el Ecuador
}

\section{Social responsibility as a labor inclusion strategy for the development of public Organization in Ecuador}

\author{
Lucy Rosero Peña \\ Universidad Tecnológica Equinoccial \\ lrosero@ute.edu.ec \\ Efraín Flores Batallas \\ Universidad Tecnológica Equinoccial \\ eflores@ute.edu.ec
}

\section{RESUMEN}

El objetivo del artículo es investigar si existe un potencial incumplimiento y falta de programación en la práctica de la Responsabilidad Social como estrategia de inclusión laboral para el desarrollo de las organizaciones del Sector Público ecuatoriano. Para ello, se realizaron indagaciones en dos instituciones públicas a efectos de contrastar la aplicación empírica de esta obligación, con las medidas prácticas requeridas, tanto a corto, como largo plazo. Los resultados apoyan la hipótesis planteada, proponiéndose un nuevo concepto, que si bien, no descarta la rutinaria y experimental acción de ayuda filántropa para solucionar un problema social, más no para preverlo, pero si garantiza la aplicación de un proceso metodológico planeado para llevar a cabo con éxito una estrategia de responsabilidad social inclusiva, encaminada a generar simbiosis con los diferentes actores sociales en pro del bien común, facilitando la medición de los efectos y su divulgación a través de reportes de sustentabilidad para conocimiento de todos los grupos de interés. Finalmente, se propone un modelo explicativo basado en instrumentos internacionales de implementación y reporte del desempeño de medidas de responsabilidad, enfocado principalmente a la inserción laboral de personas con 
discapacidad, que aportan al desarrollo institucional con su integración a los diversos procesos organizacionales, minimizando de esta manera un trato discriminatorio.

Palabras clave: Responsabilidad social, estrategia, desarrollo, sector público, organización.

\section{ABSTRACT}

The objective of the article is to investigate if there is a potential noncompliance and lack of programming in the practice of Social Responsibility as a labor inclusion strategy for the development of the Ecuadorian Public Sector organizations. For this purpose, inquiries were made in two public institutions in order to contrast the empirical application of this obligation, with the practical measures required, both in the short and long term. The results support the proposed hypothesis, proposing a new concept, which, although it does not rule out the routine and experimental philanthropic help action to solve a social problem, but not to foresee it, but it does guarantee the application of a planned methodological process to take successfully carry out an inclusive social responsibility strategy, aimed at generating symbiosis with the different social actors in favor of the common good, facilitating the measurement of the effects and its dissemination through sustainability reports for the knowledge of all stakeholders. Finally, an explanatory model based on international instruments for implementation and reporting on the performance of accountability measures is proposed, focused mainly on the labor insertion of people with special abilities, which contribute to institutional development by integrating them into the various organizational processes, minimizing This way a discriminatory treatment.

Keywords: Responsibility Social, strategy, development, public sector, organization. 


\section{INTRODUCCIÓN}

Como resultado de la evolución humana, surgen nuevos eventos, nuevas necesidades y demandas, las cuales han motivado a los entes políticos y directivos a repensar la forma de gestionar las organizaciones. Entre otras, se podría mencionar el crecimiento de la población, el incremento de la pobreza, el cambio climático, la diversidad, la inclusión y la migración.

La R.S. en la actualidad es una estrategia innovadora para gestionar las organizaciones públicas, a través de la implementación de buenas prácticas para el desarrollo y mejoramiento organizacional, enfocándose en las expectativas y necesidades de sus stakeholders, más allá de las disposiciones legales y reglamentarias, lo que permitirá el logro de la sostenibilidad a largo plazo. ${ }^{1}$

En este contexto, sobresale la dignidad humana, la solidaridad y la relación de éstas con la naturaleza y el medio ambiente, como puntos de partida para la aplicación de la R.S., seguidos de la racionalidad económica de la gestión administrativa organizacional.

La propuesta requiere un cambio apoyado en el conocimiento conceptual como elemento clave, ya que las diversas teorías administrativas condicionan las prácticas, sin tomar en cuenta que lo primero es cambiar la manera de pensar y actuar, siendo congruentes con la interpretación conceptual y la lógica de actuación.

\footnotetext{
${ }^{1}$ (Responsabilidad Corporativa y Sostenibilidad (RC\&S) Deloitte 2013)
} 
En tal virtud, se precisa diferenciar los siguientes conceptos, a fin de lograr un cambio de paradigmas en la aplicación de la R.S. que conlleve al desarrollo de las organizaciones públicas ecuatorianas, evitando controversia en su aplicación.

Tabla 1. Conceptos y cambio de paradigmas

\section{CONCEPTOS EN CONTRAPOSICIÓN DIALÉTICA}

\begin{tabular}{|c|c|c|}
\hline Filantropía & Responsabilidad Social & $\begin{array}{c}\text { Desarrollo } \\
\text { Sustentable }\end{array}$ \\
\hline $\begin{array}{ll}\text { Donaciones } & \text { de recursos } \\
\text { financieros a } & \text { un grupo de } \\
\text { personas } & \text { habitualmente } \\
\text { vulnerables } & \text { como ayuda } \\
\text { regular. } & \end{array}$ & $\begin{array}{l}\text { Acciones que generan } \\
\text { simbiosis con diferentes } \\
\text { actores sociales en pro del } \\
\text { bien común. }\end{array}$ & $\begin{array}{lr}\text { Acciones } & \text { para } \\
\text { satisfacer } & \text { las } \\
\text { necesidades } & \text { sociales y } \\
\text { mejorar la calidad de } \\
\text { vida. }\end{array}$ \\
\hline \multicolumn{3}{|c|}{ Interpretación } \\
\hline $\begin{array}{l}\text { Confusión entre filantropía y } \\
\text { caridad. La caridad mitiga } \\
\text { los problemas sociales y la } \\
\text { filantropía procura } \\
\text { resolverlos con ayuda } \\
\text { puntual, sin involucrarse. }\end{array}$ & $\begin{array}{l}\text { La donación filantrópica } \\
\text { transfiere recursos, la } \\
\text { responsabilidad social } \\
\text { invierte creando valor común } \\
\text { en el mediano y largo plazo. }\end{array}$ & $\begin{array}{l}\text { Cada sector, grupo e } \\
\text { individuo puede } \\
\text { adjudicarse } \\
\text { responsabilidad } \\
\text { particular y actuar en } \\
\text { beneficio de las actuales } \\
\text { y futuras generaciones. }\end{array}$ \\
\hline \multicolumn{3}{|c|}{ Cambio de paradigmas } \\
\hline $\begin{array}{l}\text { 1. De una posición } \\
\text { reactiva }\end{array}$ & $\begin{array}{c}\text { A una actitud solidaria } \\
\text { proactiva }\end{array}$ & $\begin{array}{l}\text { A una actitud de } \\
\text { equidad social }\end{array}$ \\
\hline $\begin{array}{l}\text { Ayuda que se brinda al } \\
\text { prójimo para solucionar un } \\
\text { problema social, más no para } \\
\text { preverlo. }\end{array}$ & $\begin{array}{l}\text { No se buscan decisiones de } \\
\text { caridad sino socios con los } \\
\text { cuáles prever los problemas } \\
\text { sociales. }\end{array}$ & $\begin{array}{l}\text { Se busca una gestión } \\
\text { proactiva del desarrollo } \\
\text { sustentable para lograr } \\
\text { una rentabilidad } \\
\text { sostenible. }\end{array}$ \\
\hline 2. De acciones dispersas & A programas permanentes & $\begin{array}{l}\text { A un modelo } \\
\text { alternativo }\end{array}$ \\
\hline $\begin{array}{l}\text { La filantropía es la actitud de } \\
\text { ayudar al prójimo con acciones } \\
\text { puntuales y específicas. }\end{array}$ & $\begin{array}{l}\text { Es una nueva visión, una } \\
\text { nueva forma de hacer las } \\
\text { cosas en beneficio del ser } \\
\text { humano y la sociedad. }\end{array}$ & $\begin{array}{l}\text { Búsqueda de respuestas } \\
\text { creativas para corregir } \\
\text { las fallas y evitar } \\
\text { nuevos problemas. }\end{array}$ \\
\hline
\end{tabular}




\begin{tabular}{|c|c|c|}
\hline $\begin{array}{c}\text { 3. De la donación sin } \\
\text { compromiso }\end{array}$ & A fondos cooperativos & $\begin{array}{c}\text { A mantener procesos } \\
\text { productivos y sociales } \\
\text { por generaciones }\end{array}$ \\
\hline $\begin{array}{l}\text { Sujetos u organizaciones que } \\
\text { desarrollan proyectos } \\
\text { solidarios. }\end{array}$ & $\begin{array}{l}\text { Cumplimiento de una } \\
\text { función no exclusivamente } \\
\text { monetaria, sino también } \\
\text { social. }\end{array}$ & $\begin{array}{l}\text { Ayudar al incremento } \\
\text { del bienestar de la } \\
\text { comunidad y a la } \\
\text { innovación } \\
\text { sostenible. }\end{array}$ \\
\hline 4. De la informalidad & $\begin{array}{l}\text { A la transparencia y } \\
\text { rendición de cuentas }\end{array}$ & $\begin{array}{l}\text { Al cumplimiento de } \\
\text { acuerdos y convenios }\end{array}$ \\
\hline $\begin{array}{l}\text { Decisiones personales, sin la } \\
\text { necesidad de rendir cuentas a } \\
\text { los demás grupos de interés. }\end{array}$ & $\begin{array}{l}\text { Medición de resultados en } \\
\text { materia de R.S. mediante } \\
\text { reportes de sustentabilidad } \\
\text { para conocimiento de los } \\
\text { grupos de interés. }\end{array}$ & $\begin{array}{l}\text { Compromiso en base de } \\
\text { la suscripción de los } \\
\text { acuerdos. }\end{array}$ \\
\hline $\begin{array}{l}\text { 5. De la acción } \\
\text { improvisada }\end{array}$ & $\begin{array}{l}\text { A la profesionalización } \\
\text { alineada a la organización }\end{array}$ & $\begin{array}{l}\text { A la innovación } \\
\text { tecnológica }\end{array}$ \\
\hline $\begin{array}{l}\text { La inversión no espera } \\
\text { beneficios, lo que la } \\
\text { diferencia de la R.S. con la } \\
\text { inversión } \\
\text { responsable. }\end{array}$ & $\begin{array}{lrr}\text { Entrega un } & \text { modelo para } \\
\text { gestionar riesgos } & \text { y } \\
\text { oportunidades } & \text { con la } \\
\text { aplicación de } & \text { políticas, } \\
\text { prácticas y } & \text { programas } \\
\text { organizacionales. } & \end{array}$ & $\begin{array}{l}\text { El avance tecnológico } \\
\text { aparece como una } \\
\text { fuente de alternativas. }\end{array}$ \\
\hline
\end{tabular}

Elaborado por los autores.

Fuentes:

- NAVAS LEORO MARÍA ALBERTINA. 2014

- RAUFFLET, LOZANO, BARRERA, GARCÍA. 2012 México.

- FERRANDO, M. y GRANERO, J. (2005)

- wikipedia.org/wiki/Filantropía

Por lo expuesto, la R.S. en las organizaciones públicas ecuatorianas requiere de una actitud solidaria y proactiva, que permita la implementación y el desarrollo de programas financiados, para gestionar riesgos y oportunidades con la aplicación de políticas y prácticas que refleje transparencia y conlleven a una permanente rendición de cuentas. 
En tal razón, lo que se pretende con el cambio de los paradigmas antes citados es vincular a la R.S. a las diferentes áreas funcionales de una organización, para pasar de ser una simple cadena de actividades a una propuesta sustentable e inclusiva.

En éste escenario, según Lenk y Jonas, ser responsable es estar dispuestos a responder a alguien o a otras instancia., Es decir, ser consecuente con las acciones y decisiones, tomando en cuenta dos factores: la voluntad de hacer algo para conseguir el bien común; y, la capacidad o habilidad física relacionada con la disponibilidad y manejo de materiales (Emmanuel Raufflet 2012).

Los antecedentes inmediatos de la R.S. sitúa el comportamiento de las grandes multinacionales americanas que a partir de 1950 comienzan a abandonar las teorías liberales más rígidas -para las que lo importante era únicamente maximizar el beneficioy comienzan a valorar adicionalmente su proyección social. (Cánovas y Mateos, 2014).

Cabe señalar, que la R.S. enlazada con el Modelo EFQM, como el compromiso voluntario que va más allá del cumplimiento de la normativa, en la atención de las necesidades de los grupos de interés, a través de un gobierno y la administración pública transparente y responsable (Cueto, 2011), es fundamental para el desarrollo del estudio.

La R.S. no puede dejar de lado el fundamento ético-filosófico. (Konrad Adenauer. 2012), a fin de cumplir con su objetivo en instituciones públicas inclusivas.

En este contexto, la R.S. de las administraciones públicas implica una gran cantidad y variedad de acciones que se apoyan en distintas metodologías, tanto a nivel macro como micro. (Artículos blog CICAP, Blog CICAP. 2016), motivando el presente estudio. 
Por otra parte, la R.S. en las instituciones públicas debe enfocar en el CÓMO hacen lo QUÉ deben hacer, entendiéndose como excelencia el diseño e implementación de sus políticas públicas alineadas con valores, transparencia, rendición de cuentas; y, diálogo social. (Charla Minvu). 2011, componentes que son parte de la propuesta.

Lenin Moreno, presentó el 24 de mayo del 2007 el programa "Ecuador sin Barreras", como política de Estado para impulsar el cumplimiento de lo que determina el Código Laboral respecto a la obligación de las empresas e instituciones en el 2007 de contratar al uno por ciento de las personas con discapacidad como parte de su nómina de empleados (Vicepresidencia de la Republica 2007).

El 25 de septiembre del 2012 se expide la Ley Orgánica de discapacidades (Asamblea 2012) puntualizando que las personas con discapacidad, son todas aquellas que a consecuencia de una o más deficiencias físicas, mentales y/o sensoriales, congénitas o adquiridas, previsiblemente de carácter permanente se ve restringida en al menos un $30 \%$ de su capacidad para realizar una actividad dentro del margen que se considera normal, en el desempeño de sus funciones.

El 29 de julio del 2009 nace la misión solidaria "Manuela Espejo" y el 20 de febrero del 2012 nace la misión solidaria "Joaquín Gallegos Lara". (Vicepresidencia de la República 2012). La primera para estudiar y registrar a las personas con discapacidad a 
nivel nacional y la segunda para cuidar a los ecuatorianos más vulnerables y olvidados, aquellos que tienen discapacidad intelectual, física severa o multi-discapacidad. ${ }^{2}$

En consecuencia, la propuesta de un modelo explicativo enfocado a la inserción laboral de personas con discapacidad en las organizaciones públicas ecuatorianas requiere de la participación y responsabilidad compartida de la alta dirección para planificar, organizar, dirigir y controlar acciones y recursos en un marco de R.S.

La implementación de la R.S. en instituciones del sector público ecuatoriano, precisa la necesidad de mantener la confianza, logrando el respeto a las reglas en un ámbito de transparencia; la ética vinculada a responder sobre el impacto de las acciones en la vida de la organización y las personas; la justicia para que las personas puedan realizarse en el ámbito laboral (Peter M. y Kramer M.R. 20016). Aplicando normas nacionales e internacionales a través de instrumentos de gestión.

Dichos instrumentos facilitan la implementación y el reporte comunicacional del desempeño de medidas de R.S. utilizando un lenguaje común. Entre los instrumentos se pueden citar: el GRI, la NORMA ISO 26000, AA1000, la Norma mexicana de Responsabilidad Social, cuyo análisis de su alcance y particularidades se presenta a continuación. Para el desarrollo de la propuesta se toma en cuenta la aplicación del GRI y la NORMA ISO 26000.

Tabla 2. Análisis del alcance de la Norma ISO 26000 y el GRI.

${ }^{2}$ http://www.vicepresidencia.gob.ec/programas/sonrieecuador/ecuadorsinbarreras/normas-deaccesibilidad.html 


\section{ISO 26000 SOBRE R.S.}

La ISO 26000 tiene como propósito generar lineamientos relacionados de manera directa con la gestión de la R.S. en las organizaciones.

\begin{tabular}{|c|c|c|c|}
\hline Filosofía & \multicolumn{2}{|r|}{ Rasgos principales } & Alcance \\
\hline $\begin{array}{l}\text { - Facilita un } \\
\text { entendimiento } \\
\text { común sobre R.S. a } \\
\text { nivel global. } \\
\text { - Sirve de enlace } \\
\text { entre los principios } \\
\text { establecidos de } \\
\text { R.S. y las diversas } \\
\text { iniciativas } \\
\text { presentadas } \\
\text { mediante } \\
\text { indicadores. }\end{array}$ & \multicolumn{2}{|c|}{$\begin{array}{l}\text { - Guía internacional reguladora, que } \\
\text { establece directrices sobre R.S. } \\
\text { - Guía para reportar avances cualitativos, } \\
\text { no cuantitativos en materia de R.S. y } \\
\text { sustentabilidad. } \\
\text { - No es una norma de sistema de gestión, } \\
\text { se queda a nivel de lineamientos y } \\
\text { recomendaciones. } \\
\text { - Se puede implementar en cualquier tipo } \\
\text { de organización, independientemente de } \\
\text { su actividad, ubicación y tamaño. }\end{array}$} & $\begin{array}{l}\text { - Establece recomendaciones } \\
\text { que permiten implementar } \\
\text { prácticas de R.S. } \\
\text { - Toma en cuenta la gestión de } \\
\text { la política institucional, los } \\
\text { derechos humanos, las } \\
\text { prácticas laborales, el } \\
\text { ambiente, las operaciones } \\
\text { justas, los consumidores y el } \\
\text { desarrollo de la comunidad. }\end{array}$ \\
\hline \multicolumn{4}{|c|}{ Indicadores GRI } \\
\hline \multicolumn{2}{|l|}{ Filosofía } & Rasgos principales & Alca \\
\hline \multicolumn{2}{|c|}{$\begin{array}{l}\text { Apoya con lineamientos } \\
\text { indicadores y documentos } \\
\text { para implementar medidas } \\
\text { de R.S. y realizar reportes } \\
\text { sobre sustentabilidad, } \\
\text { comparables entre } \\
\text { organizaciones públicas y } \\
\text { privadas. }\end{array}$} & $\begin{array}{l}\text { - Aplicable a pequeñas y } \\
\text { medianas organizaciones. } \\
\text { - En la actualidad es la guía de } \\
\text { mayor uso. } \\
\text { - No se presenta como un } \\
\text { modelo de gestión, lo que } \\
\text { limita la obtención de una } \\
\text { certificación externa. }\end{array}$ & $\begin{array}{l}\text { - Medición del desempeño } \\
\text { mediante indicadores en la } \\
\text { implementación del triple } \\
\text { balance: económico, ambiental } \\
\text { y social. }\end{array}$ \\
\hline
\end{tabular}

Elaborado por los autores

\section{Fuentes:}

- NAVAS LEORO MARÍA ALBERTINA. 2014.

- RAUFFLET, LOZANO, BARRERA, GARCÍA. 2012.

- FERRANDO, M. y GRANERO, J. (2005)

\section{METODOLOGÍA}

En el estudio se aplica la investigación cualitativa, "que narra, describe, o explica minuciosamente lo que está sucediendo en un momento dado” (Garcés, 2000, p.75), siendo necesario comprender a "partir de la explicación análoga de la realidad", (Mendoza, V, p.22), en este caso se presenta la necesidad de conocer la aplicación de 
procesos de R.S. como estrategia de inclusión laboral en las organizaciones seleccionadas.

Con la aplicación de la investigación cualitativa es factible manejar los estudios de caso, ya que se "analiza una unidad para responder al planteamiento del problema, probar la hipótesis y desarrollar alguna teoría”. (Hernández, Fernández y Baptista 2010. p.163), y en consecuencia bajo el análisis y razonamiento particular de cada organización seleccionada determinar las condiciones de aplicabilidad de la R.S. como estrategia de inclusión laboral.

Para la selección de los casos se aplicó un estudio teórico, no estadístico (Garcés, 2000), obteniéndose una muestra, la misma que facilita la obtención de prácticas de aprendizaje para el desarrollo de una realidad que pretende la aplicación de la R.S. inclusiva a partir de la experiencia.

Para el estudio teórico de caso se utilizaron primer lugar, fuentes secundarias basadas en información documental de cada organización seleccionada. En segundo lugar, se realizó una entrevista grupal, aplicando la técnica del Brainstorming al personal de ejecutivos, como fuente de información principal, cuyo resultado permite el diagnóstico del problema y sus posibles causas, garantizando la generación de nuevas ideas para la solución del problema identificado, asegurando la calidad en las decisiones, el compromiso en el trabajo de los involucrados, y la responsabilidad compartida, exigencias indispensables para llevar a efecto el modelo explicativo de este estudio. 
En tercer lugar, se procede a graficar el diagrama causa - efecto con las ideas más representativas receptadas a través del Brainstorming, identificando las causas raíces y las causas secundarias, a fin de establecer las posibles soluciones al problema identificado.

En cuarto lugar, se identifica mediante la Matriz de Multivotación los criterios más reveladores sobre posibles soluciones y su puesta en práctica. En esta fase exploratoria los descubrimientos permiten llegar a definir un escenario que soporte la certificación del estudio.

Partiendo de la rúbrica de la entrevista grupal de las ideas calificadas del 1 al 3 , se selecciona aquellas que han alcanzado la calificación de 1, en cada uno de los aspectos vinculados con el tema central de la investigación. En el desarrollo se presenta el resultado unificado del análisis de los dos casos de estudio. 
DESARROLLO

Tabla 3. Problema y causas que lo generan.

\begin{tabular}{|c|c|}
\hline \multicolumn{2}{|r|}{ ORGANIZACIONES SELECCIONADAS } \\
\hline \multicolumn{2}{|r|}{$\begin{array}{l}\text { Institución 1.- Institución líder desde hace } 35 \text { años en el financiamiento de inversión } \\
\text { pública, brindando servicios financieros y no financieros. }\end{array}$} \\
\hline \multicolumn{2}{|r|}{$\begin{array}{l}\text { Institución 2- Empresa Pública líder desde los años ochenta en la provisión del } \\
\text { servicio de energía eléctrica, en la ciudad de Quito. }\end{array}$} \\
\hline \multicolumn{2}{|r|}{$\begin{array}{l}\text { IDENTIFICACIÓN DEL PROBLEMA Y POSIBLES CAUSAS APLICANDO EL } \\
\text { BRAINSTORMING }\end{array}$} \\
\hline \multicolumn{2}{|r|}{$\begin{array}{l}\text { PARTICIPANTES: Grupo multidisciplinario que ostenta funciones de dirección en } \\
\text { las dos organizaciones. }\end{array}$} \\
\hline \multicolumn{2}{|r|}{$\begin{array}{l}\text { PROBLEMA: Ausencia de un modelo socialmente inclusivo de R.S. que oriente a la } \\
\text { gestión, control, comunicación y toma de decisiones, con calidad y enfoque de } \\
\text { servicio. }\end{array}$} \\
\hline No. & POSIBLES CAUSAS \\
\hline 1 & Inadaptabilidad en nuevas instalaciones de trabajo. \\
\hline 2 & $\begin{array}{l}\text { Problemas tecnológicos por fallas no previstas en el manejo de las nuevas } \\
\text { tecnologías implementadas. }\end{array}$ \\
\hline 3 & $\begin{array}{l}\text { Problemas de comunicación por efectos jerárquicos, derivados de la } \\
\text { estructura vertical vigente. }\end{array}$ \\
\hline 4 & $\begin{array}{l}\text { Recorte del presupuesto administrativo por políticas de austeridad aplicadas } \\
\text { en el sector público. }\end{array}$ \\
\hline 5 & $\begin{array}{l}\text { Falta de un proyecto de R.S. en el ámbito de la inserción laboral de personas } \\
\text { con discapacidad. }\end{array}$ \\
\hline 6 & $\begin{array}{l}\text { Ausencia de políticas para la selección e inserción laboral de personas con } \\
\text { discapacidad. }\end{array}$ \\
\hline 7 & $\begin{array}{l}\text { Falta de políticas de seguridad y salud ocupacional con repercusión en el } \\
\text { desempeño laboral. }\end{array}$ \\
\hline 8 & $\begin{array}{l}\text { Falta de preparación del personal en la aceptación de personas con } \\
\text { discapacidad. }\end{array}$ \\
\hline
\end{tabular}




\begin{tabular}{|c|l|}
\hline 9 & $\begin{array}{l}\text { Incremento de riesgos de trabajo por la falta de un proceso especializado } \\
\text { para la selección de personal con discapacidad. }\end{array}$ \\
\hline 10 & $\begin{array}{l}\text { Falta de un proceso comunicacional que fomente una cultura de } \\
\text { responsabilidad social basada en la inclusión. }\end{array}$ \\
\hline 11 & Falta de descriptores de cargos específicos para personas con discapacidad. \\
\hline 12 & $\begin{array}{l}\text { Falta de indicadores de desempeño social, en cuestiones laborales, de la Guía } \\
\text { para la elaboración de memorias de sostenibilidad GRI. }\end{array}$ \\
\hline 13 & $\begin{array}{l}\text { Falta de políticas y estándares relacionados con la inserción del grupo de } \\
\text { interés con discapacidad, según el estándar de aseguramiento AA 1000. }\end{array}$ \\
\hline $\begin{array}{l}\text { Facilitadores: Lucy Rosero y Efraín Flores } \\
\text { Fecha: } 24 \text { de septiembre del 2017 } \\
\text { Lugar: Quito - Ecuador }\end{array}$
\end{tabular}

Elaborado por los autores 
APLICACIÓN DEL DIAGRAMA DE CAUSA-EFECTO PARA GRAFICAR EL PROBLEMA, LAS CAUSAS RAÍCES Y SECUNDARIAS IDENTIFICADASEN LA SESIÓN DE BRAINSTORMING

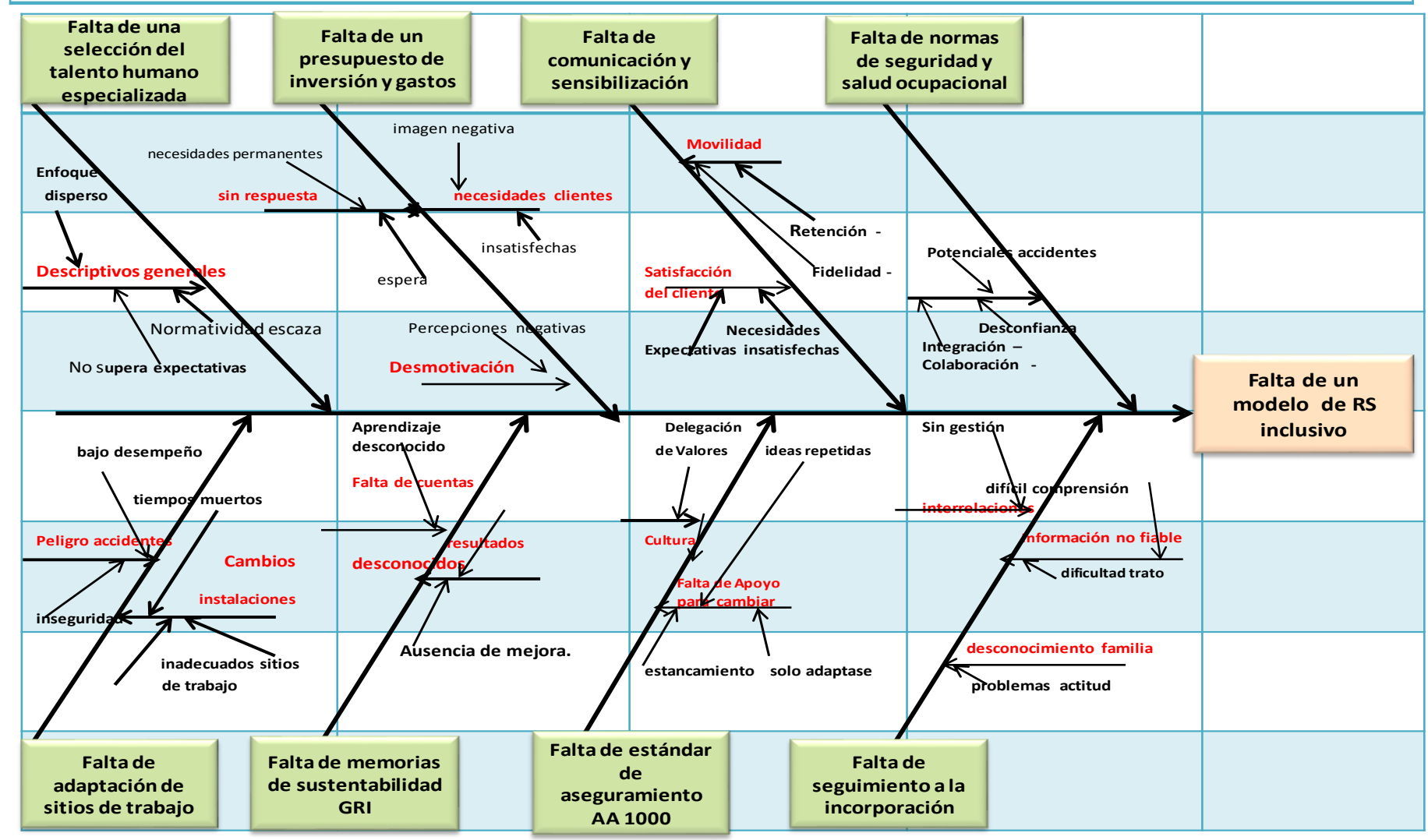


Tabla 4. MATRIZ DE MULTIVOTACIÓN APLICANDO EL BRAINSTORMING

PARTICIPANTES: Grupo multidisciplinario que ostenta funciones de dirección.

PROBLEMA: Ausencia de un modelo socialmente inclusivo de R.S. que oriente la gestión, control, comunicación y toma de decisiones oportunas, con calidad y con enfoque de servicio.

\begin{tabular}{|c|c|c|c|c|}
\hline \multirow[t]{2}{*}{ No. } & \multirow{2}{*}{ POSIBLES SOLUCIONES } & \multicolumn{3}{|c|}{ PRIORIDADES } \\
\hline & & 1 & 2 & 3 \\
\hline 1 & $\begin{array}{l}\text { Delimitar las responsabilidades administrativas en función de los } \\
\text { programas que ejecuta la organización. }\end{array}$ & & $\mathrm{X}$ & \\
\hline 2 & Facilitar la selección y control del talento humano. & $\mathrm{X}$ & & \\
\hline 3 & $\begin{array}{l}\text { Definir el grado de autonomía de la aplicación de la R.S. con relación } \\
\text { a las unidades funcionales. }\end{array}$ & $\mathrm{X}$ & & \\
\hline 4 & $\begin{array}{l}\text { Aplicar técnicas de selección especializada para personal con } \\
\text { discapacidad. }\end{array}$ & $\mathrm{X}$ & & \\
\hline 5 & Definir indicadores de gestión. & & $\mathrm{X}$ & \\
\hline 6 & Tratar a tiempo las formas de discriminación. & $\mathrm{X}$ & & \\
\hline 7 & $\begin{array}{l}\text { Acompañamiento que favorezca el equilibrio trabajo, persona y } \\
\text { familia. }\end{array}$ & $\mathrm{X}$ & & \\
\hline 8 & Inserción estratégica de personas con discapacidad. & $\mathrm{X}$ & & \\
\hline 9 & Identificar las características del cargo y el entorno para el desempeño. & $\mathrm{X}$ & & \\
\hline 10 & $\begin{array}{l}\text { Identificar las condiciones de las personas y las características del } \\
\text { cargo. }\end{array}$ & $\mathrm{X}$ & & \\
\hline 11 & Definir medios de reclutamiento. & $\mathrm{X}$ & & \\
\hline 12 & Desarrollar habilidades y competencias. & $\mathrm{X}$ & & \\
\hline 13 & Proveer de un presupuesto de inversiones y gastos. & $\mathrm{X}$ & & \\
\hline 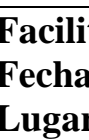 & $\begin{array}{l}\text { ores: Lucy Rosero y Efraín Flores } \\
4 \text { de septiembre del } 2017 \\
\text { uito - Ecuador }\end{array}$ & & & \\
\hline
\end{tabular}

Elaborado por los autores 


\section{RESULTADOS Y DISCUSIÓN}

Del análisis comparativo de los casos de estudio, es factible establecer varios factores que influyen en los resultados de corto y largo plazo en cada organización seleccionada.

A pesar de las diferencias propias de cada organización éstas coinciden en la necesidad de utilizar instrumentos de medición para la programación, control y reporte de la R.S. inclusiva. Las principales diferencias se presentan en la forma de utilizarlos para la solución del problema descrito.

En consecuencia bajo el análisis y razonamiento particular de cada organización seleccionada se podrán determinar las condiciones de aplicabilidad de la R.S. como estrategia de inclusión laboral.

A futuro se aspira lograr la implementación del modelo, el mismo que permitirá el real posicionamiento de las organizaciones en el sector de su competencia, bajo normas internacionales de R.S., facilitando además el control del cumplimiento de los indicadores de cada uno de sus componentes.

De tal forma, la aplicación y seguimiento de la propuesta, cambiará el enfoque filántropo convencional, ya que en la práctica es una estrategia de negocio y no el resultado de la práctica de una visión parcializada de los directivos.

Además, se determina que carecen de procedimientos que les permita la aplicación de la R.S. en toda su magnitud, lo que les limita la participación inclusiva en la sociedad. 
El modelo sugerido como respuesta al análisis de caso, permitirá la ejecución de procedimientos que viabilicen la inserción laboral de personas discapacitadas, que identifique al candidato apto a las necesidades específicas del cargo y de ser posible a las necesidades generales de la organización.

\section{CONCLUSIONES}

Las organizaciones analizadas no aplicaron los instrumentos estratégicos de planeación y medición de la R.S. GRI y la Norma ISO 26000, generado resultados negativos tanto a corto, como a largo plazo. La recomendación en este sentido estaría dirigida a la alta dirección para que se preparen en el conocimiento del alcance y objetivos que éstas implícitamente persiguen. Tabla 1., y de los insumos que deben tener para su desarrollo. Tabla 2.

En este contexto, es recomendable que se planifique de acuerdo a los objetivos específicos de cada organización y que la alta dirección se encuentre preparada para ejecutar, verificar y actuar sobre los resultados y las experiencias alcanzadas.

Finalmente, con todos los antecedentes se valida la presentación de un modelo explicativo, Grafico 1., que permite integrar en una secuencia lógica la aplicación de los instrumentos de R.S. GRI y la Norma ISO 26000.

Grafico 1. Modelo explicativo 


\section{PROPUESTA PARA IMPLEMENTAR UN MODELO DE R. S. INCLUSIVO EN ORGANIZACIONES DEL SECTOR} PÚBLICO ECUATORIANO

\begin{tabular}{|c|c|c|c|c|}
\hline \multicolumn{5}{|c|}{ 1.1 Presentación del Modelo sugerida como repuesta al análisis de caso. } \\
\hline \multicolumn{5}{|c|}{$\begin{array}{l}\text { Desde la R.S. puede llevarse a cabo con éxito este modelo siguiendo un proceso metodológico fundamentado en el conocimiento conceptual y en } \\
\text { la realidad práctica. }\end{array}$} \\
\hline Base legal & Objetivo general & Insumos & Objetivos específicos & Resultados \\
\hline $\begin{array}{l}\checkmark \text { Art.3 Reglamento General de la Ley } \\
\text { Reformatoria de la Ley de } \\
\text { Discapacidades. (2013) } \\
\text { La persona discapacitada es toda aquella } \\
\text { que, como consecuencia de una o más } \\
\text { deficiencias físicas, mentales y/o } \\
\text { sensoriales, congénitas o adquiridas, } \\
\text { previsiblemente de carácter permanentes } \\
\text { se ve restringida en al menos un } 30 \% \text { su } \\
\text { capacidad para realizar una actividad } \\
\text { dentro del margen que se considera } \\
\text { normal. } \\
\checkmark \\
\text { Ley Orgánica de Discapacidades (2012) } \\
\text { Las empresas e instituciones disponen de } \\
\text { esta fuerza laboral (2010), que según la } \\
\text { Misión Manuela Espejo hay } 2.7 \% \text { de } \\
\text { personas con capacidades especiales en el } \\
\text { Ecuador, y se necesita que al menos el } 4 \% \\
\text { de la nómina de las empresas incluyan a } \\
\text { este tipo de personas. }\end{array}$ & \begin{tabular}{|l}
$\checkmark$ \\
\multicolumn{2}{l}{ La R.S. aplicada como } \\
estrategia \\
desarrollo de \\
organizaciones den \\
sector público \\
ecuatoriano, irá más \\
allá del cumplimiento \\
de las leyes, \\
reglamentos y normas, \\
realizando r una \\
contribución activa y \\
voluntaria \\
mejoramiento social y \\
económico.
\end{tabular} & $\begin{array}{l}\text { Disposición de la } \\
\text { alta gerencia no } \\
\text { solo para el } \\
\text { cumplimiento } \\
\text { legal, sino como } \\
\text { puesta en marcha } \\
\text { de una iniciativa de } \\
\text { R.S. inclusiva. }\end{array}$ & 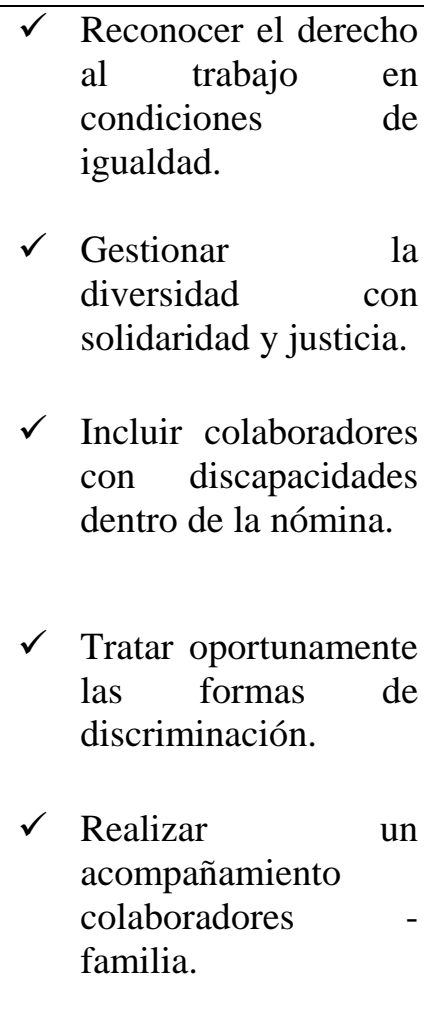 & 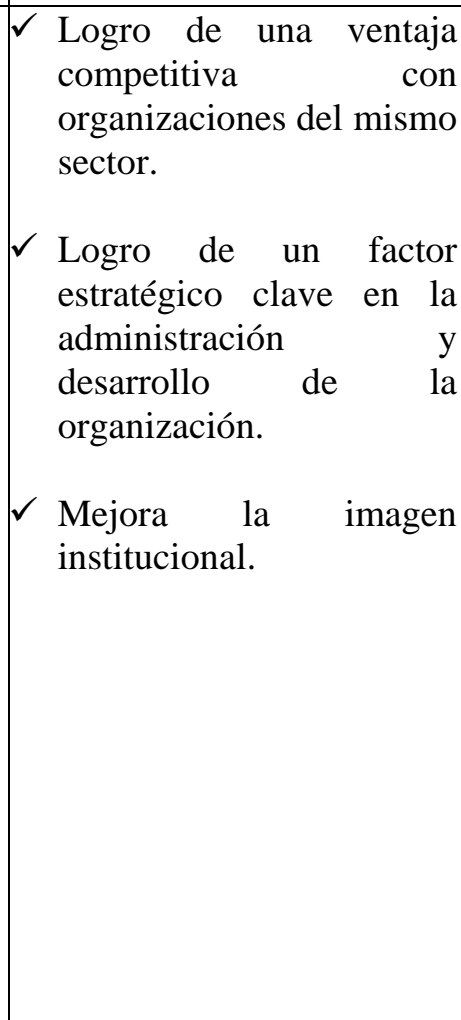 \\
\hline
\end{tabular}




\begin{tabular}{|c|}
\hline $\begin{array}{l}\text { Misiones solidarias: } \\
\text { "Manuela Espejo" } \\
\text { Registra a las personas con discapacidad a } \\
\text { nivel nacional. } \\
\text { "Joaquín Gallegos Lara" } \\
\text { Cuida de los ecuatorianos más vulnerables } \\
\text { y olvidados que tienen discapacidad } \\
\text { intelectual, física severa o multi- } \\
\text { discapacidad. }\end{array}$ \\
\hline
\end{tabular}

Elaborado: por los autores

- LEY ORGÁNICA DE DISCAPACIDADES. 2012

- leY ORGÁNICA DE DISCAPACIDADES. 2013

- PRIMER REgISTRO NACIONAL SOBRE INCLUSIÓN LABORAL DE PERSONAS CON DISCAPACIDAD (2009 al 2009) 


\begin{tabular}{|c|c|c|c|c|c|}
\hline \multicolumn{6}{|c|}{$\begin{array}{l}\text { 1.2 Planificación de la R.S. como estrategia de desarrollo en organizaciones del Sector Público ecuatoriano, aplicando los Indicadores } \\
\text { GRI de triple balance: ambiental, económico y social. }\end{array}$} \\
\hline \multicolumn{6}{|c|}{$\begin{array}{l}\text { COMPONENTE AMBIENTAL } \\
\text { OBJETIVO 1: PREPARACIÓN DEL ENTORNO }\end{array}$} \\
\hline Debilidad o necesidad & Estrategia & Actividades/Tareas & Evidencias & Fecha & Responsables \\
\hline 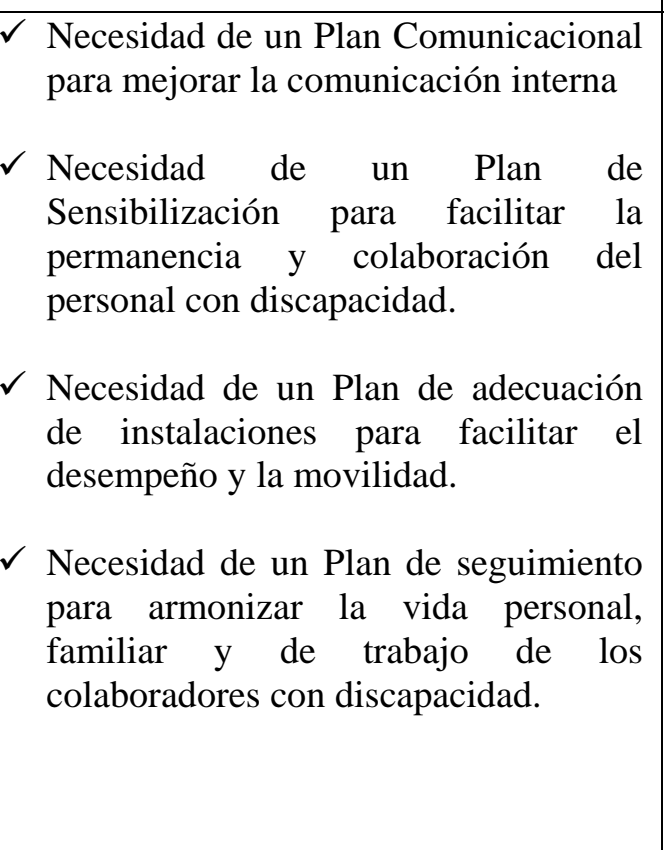 & 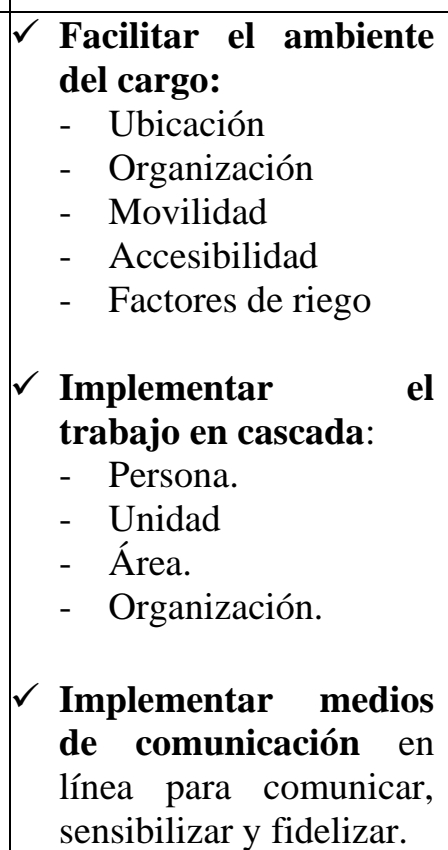 & 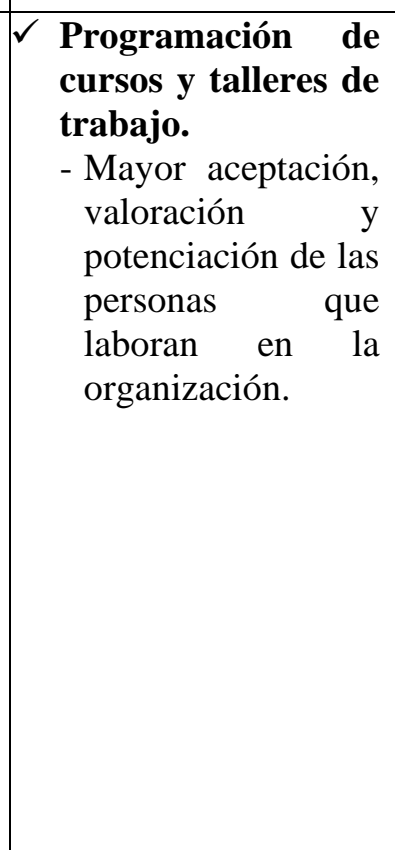 & 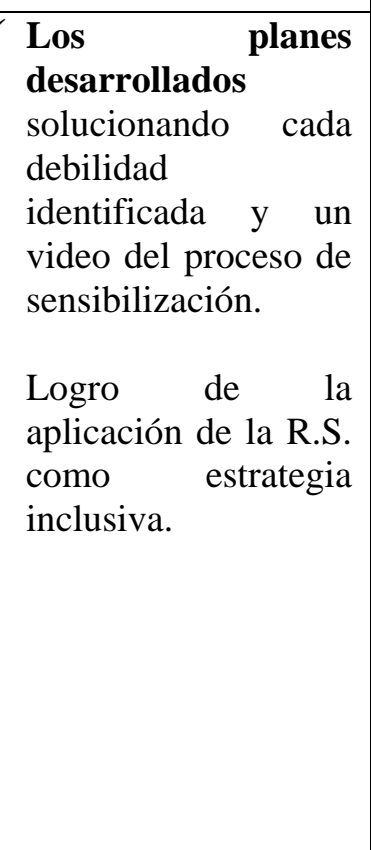 & $\begin{array}{c}04 / 12 / 2017 \\
\text { al } \\
30 / 03 / 2018\end{array}$ & \begin{tabular}{|ll}
$\checkmark$ & Dirección \\
& Recursos \\
& Humanos. \\
& \\
$\checkmark$ & Unidad de \\
& Bienestar \\
& Social. \\
& \\
$\checkmark$ & Dirección de de \\
& Comunicación \\
& Interna.
\end{tabular} \\
\hline \multicolumn{6}{|c|}{ COMPONENTE SOCIAL } \\
\hline \multicolumn{6}{|c|}{ OBJETIVO 2: PREPARACIÓN DEL PROCESO DE SELECCIÓN } \\
\hline Debilidad o necesidad & Estrategia & Actividades/Tareas & Evidencias & Fecha & Responsables \\
\hline
\end{tabular}




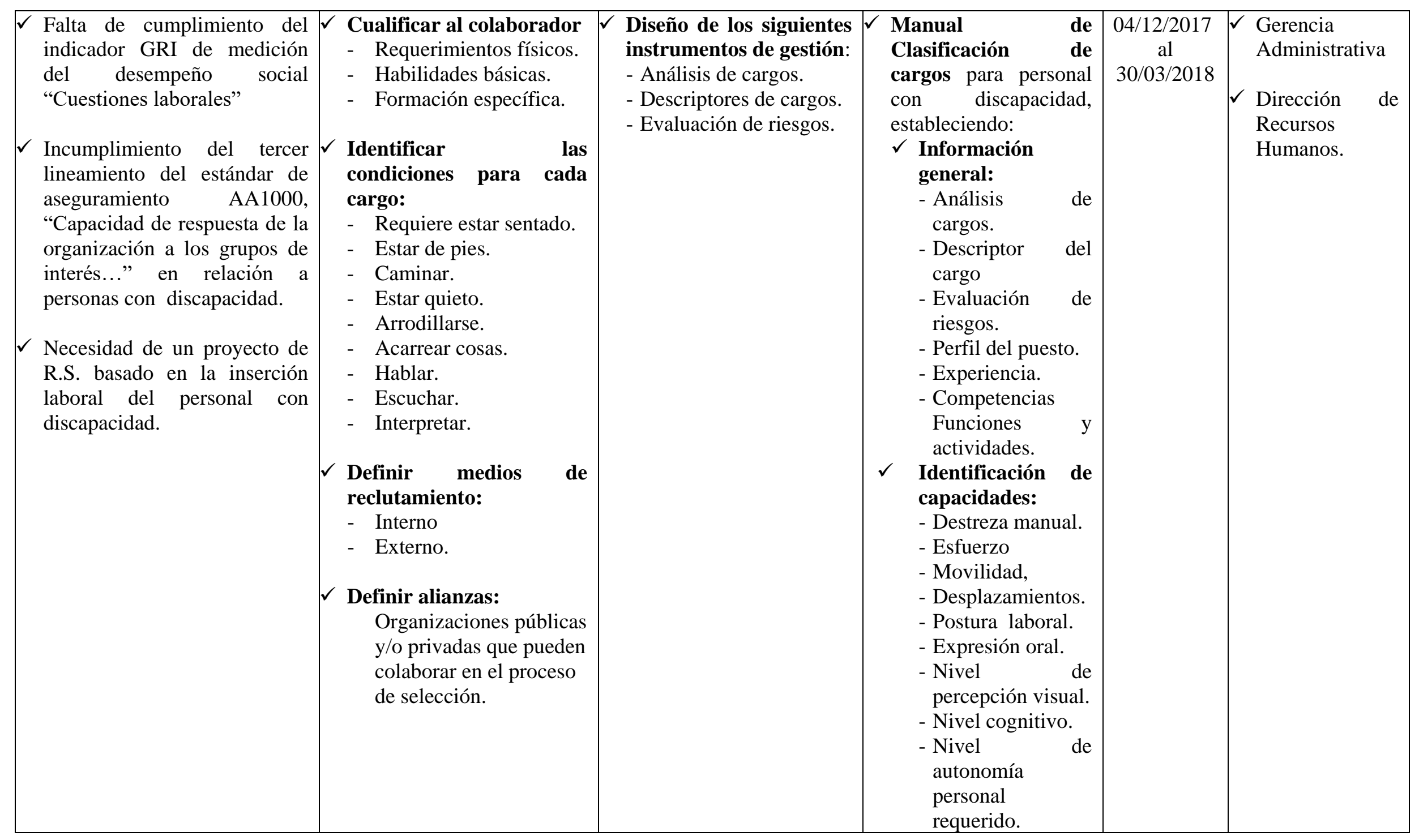




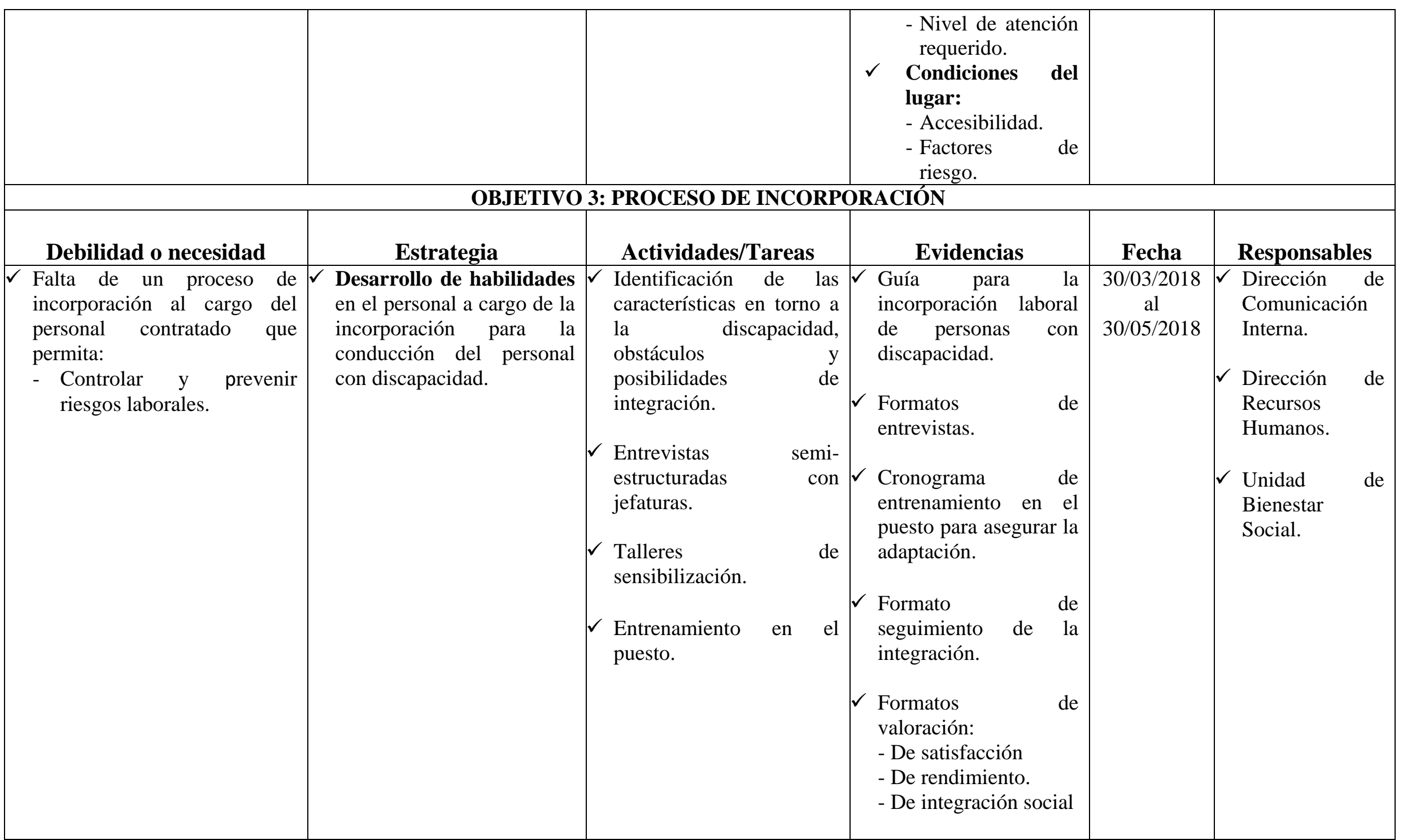


COMPONENTE ECONÓMICO

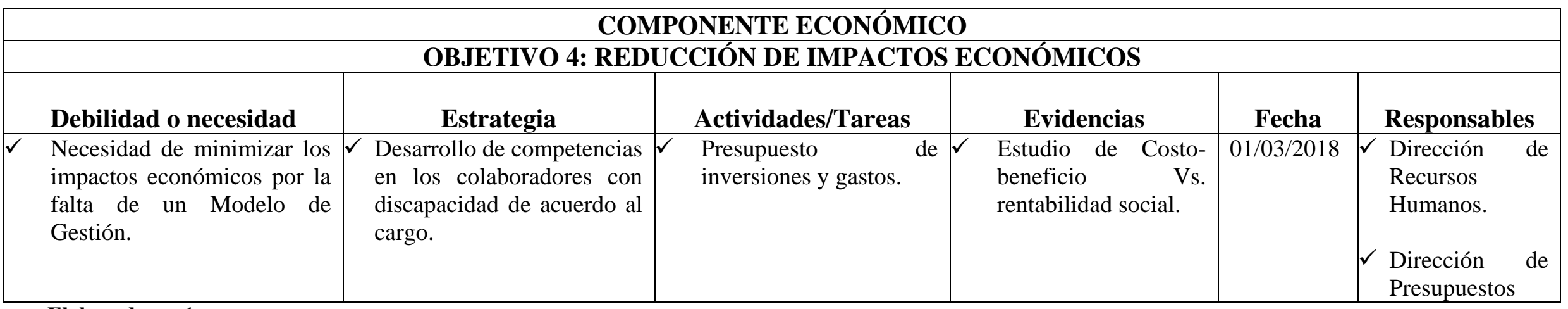

Elaborado por los autores

Fuente: RAUFFEL, LOZANO, BARRERA, GARCÍA DE LA TORRE. 2013 


\section{BIBLIOGRAFÍA}

\section{Libros:}

- CÁNOVAS, J. Y MATEOS, J., (2014), Responsabilidad Social Corporativa: más que un modelo de gestión, un reto para las Administraciones Públicas, Murcia, España: Escuela de Formación e Innovación de Murcia, 9-16, 23-33, 45-46, 78, 82-84.

- CUETO, C. Y DE LA CUESTA, M., (2011), Responsabilidad Social en la Administración Pública y Modelo de Excelencia en la Gestión (EFQM), Madrid, España: CátedraTelefónica de Responsabilidad Corporativa y Sostenibilidad, UNED, $1-4$.

- FERRANDO, M. y GRANERO, J. (2005) Calidad total: Modelo EFQM de excelencia "FC Editorial (Fundación Confederal)" Madrid

- LEY ORGÁNICA DE DISCAPACIDADES. Suplemento -- Registro Oficial N. o 796 -martes 25 de septiembre del 2012.

- LEY ORGÁNICA DE DISCAPACIDADES. Segundo Suplemento -- Registro Oficial $\mathrm{N}^{\mathrm{o}} 145$-- martes 17 de diciembre del 2013

- NAVAS LEORO MARÍA ALBERTINA. Texto - Guía Maestría Gestión Empresarial. Responsabilidad Social Empresarial. Editora. Cía. Ltda. 2014. Loja.

- RAUFFLET, LOZANO, BARRERA, GARCÍA. Responsabilidad Social Empresarial. Pearson. 2012. México.

- KONRAD ADENAUER (Responsabilidad Social Empresarial: Una respuesta ética ante los desafíos globales. Cátedra de Economía Social del Mercado.. Primera edición 2012.)

\section{Referencias}

CHARLA MINVU. Responsabilidad Social en instituciones públicas.. Superintendencia de Seguridad Social (SUSESO).Macarena Candia. Santiago, 18 de enero de 2011.

EMMANUEL RAUFFLET, JOSÉ FÉLIX LOZANO AGUILAR, ERNESTO BARRERA LUQUE, CONSUELO GARCÍA DE LA TORRE. Responsabilidad empresarial. Editorial Pearson México 2012, Pg., 22 
La responsabilidad social en la administración pública. Universidad Pontificia Comillas. Madrid. 2013

La responsabilidad social en el sector público. El 16 septiembre, 2016 / Artículos

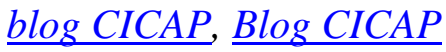

PORTER M, Y KRAMER M.R. (2006) "Strategy \& Society: The link between competitive advantage and Corporate Social Responsibility, Harvard Business Review. Vol.84.Issue.12. pp. $78-92$

Primer registro nacional sobre inclusión laboral de personas con discapacidad (Pichincha - noviembre del 2009 al 15 de diciembre del 2009).

\section{Páginas web}

- wikipedia.org/wiki/Filantropía

- http://www.vicepresidencia.gob.ec/programas/sonrieecuador/ecuadorsinbarreras/norm as-de-accesibilidad.html

\section{Tablas:}

Tabla 1. Conceptos y cambio de paradigmas

Tabla 2. Análisis del alcance de la Norma ISO 26000 y el GRI

Tabla 3. Organizaciones seleccionadas para el estudio de caso 